\title{
Investigation of The Effects of The Toll-Like \\ Receptor 4 Pathway on Immune Checkpoint Vista in Pancreatic Cancer
}

\section{Kübra Sena Bas Topcu}

Bartın Üniversitesi Fen Fakültesi: Bartin Universitesi Fen Fakultesi

Emine Nedime KORUCU ( $\boldsymbol{D}$ enkorucu@erbakan.edu.tr)

Necmettin Erbakan University Faculty of Science: Necmettin Erbakan Universitesi Fen Fakultesi https://orcid.org/0000-0001-7034-4130

\section{Esma Menevse}

Selçuk University Faculty of Medicine: Selcuk Universitesi Tip Fakultesi

\section{Nadir Kocak}

Selçuk University Faculty of Medicine: Selcuk Universitesi Tip Fakultesi

\section{Tugce Duran}

KTO Karatay Universitesi

\section{Research Article}

Keywords: Pancreatic ductal adenocarcinoma, siRNA, TLR4, VISTA, Immunotherapy

Posted Date: December 9th, 2021

DOI: https://doi.org/10.21203/rs.3.rs-1064217/v1

License: (1) This work is licensed under a Creative Commons Attribution 4.0 International License.

Read Full License

Version of Record: A version of this preprint was published at Investigational New Drugs on February 3rd, 2022. See the published version at https://doi.org/10.1007/s10637-021-01209-z. 


\section{Abstract}

Pancreatic ductal adenocarcinoma (PDAC) is one of the most common malignant tumors of the pancreas. Preclinical studies show that it evades the immune system with immune checkpoints and promotes tumor development. V-domain Ig suppressor of T cell activation (VISTA) is a new immunecheck point from the B7 family and is highly expressed in cancer cells. Overexpression of toll like receptor 4 (TLR4) in pancreatic adenocarcinoma is associated with inducing tumorigenesis, tumor growth and resistance to chemotherapy. Naloxone is an opioid and inhibits TLR4-ligand association. In this study, we investigated the connection of TLR4 and downstream pathway with immune-check point VISTA in pancreatic cancer proliferation. We first collected pancreatic cancer-related datasets using the GEPIA2 and UALCAN databases. Based on the data obtained the effect of different concentrations and incubation times of Naloxone were used on PANC-1 cells proliferation. Combination of Naloxone and VISTA-siRNA were applied, and effect of both Naloxone and combined reatment on TLR4, Interleukin 1 receptor associated kinase 4 (IRAK4) and VISTA gene expression were analysed in pancreatic cancer cells. As a result of analysis with Quantitative Real-Time Polymerase Chain Reaction (qRT-PCR), gene expression levels of TLR4, IRAK4 and VISTA were significantly suppressed and cell proliferation was significantly reduced. We found that administration of Naloxone and VISTA-siRNA in combination to PDAC cells suppressed signaling. Therefore, we considered that the relationship between VISTA and TLR4 signaling pathways and the other possible associated signal molecules may be an important marker in determining the response of immune checkpoint inhibitors in cancer treatment.

\section{Introduction}

Cancer occurs as a result of continuous cell proliferation upon the damage of genetic material by transforming normal cells into malignant over time with the effect of mutations [1]. According to GLOBOCAN data, approximately 19.3 million new cancer cases and roughly 10 million cancer-related deaths were detected worldwide in 2020 [2]. Pancreatic ductal adenocarcinoma (PDAC) is one of the most common malignant tumors of the pancreas [3]. The cause of this very common cancer type is not known yet, and risk factors have been determined asage, smoking, obesity, diabetes, chemical carcinogens (gasoline, pesticides), gender, Helicobacter pylori infection, chronic pancreatitis and family history. According to GLOBOCAN 2020 estimates, pancreatic cancer is the 12th most common cancer, causing 466003 deaths and 495773 new cases worldwide [2]. Since pancreatic cancer does not show any symptoms in the early stages, it spreads rapidly and therefore it is very difficult to diagnose. Due to the highly heterogeneous and complex nature of pancreatic cancer, it is thought that the development of targeted and combined treatment strategies may increase the survival rate [4].

Cancer cells manage to protect themselves from immune system cells by expressing or stimulating immune checkpoints that regulate immunity in the cell membrane for suppression of autoimmunity. The $\mathrm{V}$-domain immunoglobulin suppressor of $\mathrm{T}$ cell activation ( $\mathrm{V}$-set immunoregulatory receptor; VISTA) is a Type 1 transmembrane protein that functions as a negative immune checkpoint molecule. It is locazited on CD4+ and CD8+ T helper cells as an inhibitory receptor and it strongly suppresses $T$ cell activation and 
cytokine production [5]. Toll was first identified as the gene encoding the transmembrane protein in the species Droshopilla melanogaster [6]. With later studies, the homolog of the protein encoded by the toll gene was detected in mammals and named as toll-like receptor (TLR) [7]. TLR4, a member of the TLR family, is known as a cell membrane receptor that activates with both LPS (Lipopolysaccarides) and DAMPs (Damage-associated molecular pattern) and causes proinflammatory cytokine synthesis. It is expressed in innate immune cells and many tumor cells. It is especially involved in triggering tumor formation, tumor growth, inducing apoptosis and resistance to chemotherapy [8]. RNA interference (RNAi) is known as a highly specific approach used to suppress or silence gene expression. It has significant potential for the treatment of various pathological conditions such as cardiovascular diseases, viral infections and cancer. In this method, non-coding RNA molecules inhibit target gene expression or translation in a sequence-specific manner [9].

In this study, TLR4 gene expression, which plays an important role in the progression of pancreatic cancer cell line (PANC-1), was performed to be silenced with the TLR4 antagonist Naloxone. At the same time, it was aimed to specifically silence the negative immune checkpoint VISTA gene expression with siRNA and subsequently to evaluate the relationship of TLR4 signaling pathway with VISTA. VISTA encoded by the VSIR gene and TLR4 signaling pathways showed that both proteins are associated with the NF-KB signaling pathway (https://www.genecards.org, Accessed 08.05.2021). However, potential links between VISTA and TLR4 expression in pancreatic cancer have not yet to be discovered. Therefore, we think that findings of the present study will contribute to the literature about treatment of pancreatic cancer.

\section{Materials And Methods}

\section{GEPIA2 database analysis}

GEPIA2 (Gene Expression Profiling Interactive Analysis 2) database (http://gepia2.cancer-pku.cn/) is a quotable resource for gene expression analysis based on tumor and normal samples from the TCGA and GTEx databases [10]. It was used to compare the gene expression levels of TLR4, IRAK4 and VISTA in PANC-1 compared to normal cells. The $p$-value was generated using Student's t-test and was considered significant for $<0.05$. $\left|\log _{2} \mathrm{FC}\right|>1$ indicates the expression of a gene between cancer and normal cells.

\section{Ualcan Database Analysis}

UALCAN (http://ualcan.path.uab.edu/index.html) is an effective web resource that can be analyzed based on relevant cancer data using the TCGA database. In this study, UALCAN was used to observe the association of target gene expression with patient survival [11]. The $p$-value was generated using Student's t-test and was considered significant for $<0.05$.

\section{Cell Culture}


The American Type Culture Collection provided the human pancreatic cancer PANC-1 cell line and the human umbilical vein endothelial cell line (HUVEC) cells used in this obtain (ATCC, USA). PANC-1 cells were cultured in Dulbecco's Modified Eagle's medium (DMEM; Gibco; Thermo Fisher Scientific, Inc., Waltham, MA, USA) supplemented with 10\% fetal bovine serum (Hyclone; FBS), penicillin $(100 \mathrm{U} / \mathrm{mL})$, and streptomycin $(100 \mathrm{~g} / \mathrm{mL})$ at $37^{\circ} \mathrm{C}$ in an incubator with $5 \% \mathrm{CO} 2$. Cells were grown in antibiotic-free medium for transfection studies.

\section{Naloxone Treatment Of Panc-1 Cells}

Naloxone drug known as TLR4 antagonist was purchased from Hameln Pharma Plus (GmbH, Germany). It was prepared at different concentrations $(150-400 \mu \mathrm{M})$ and cell viability analysis (MTT analysis) was performed and the IC50 value was determined. In the next step, qRT-PCR was performed to detect the effects of Naloxone on TLR4, IRAK 4 and VISTA gene expressions.

\section{Sirna Treatment}

For transfections, $25 \times 10^{3}$ cells were seeded per well (in a 6-well plate) in antibiotic-free medium and cells were incubated until they were confluent.

siRNAs specific for target genes were designed and purchased by Dharmacon (GE Healthcare, USA). VISTA, GAPDH and negative control siRNA were used in the study. siRNAs were supplied in powder form and prepared according to the Dharmacon protocol.

To determine the optimum percent silencing of siRNA, it was determined at different concentrations of 5 $\mathrm{nM}, 10 \mathrm{nM}, 25 \mathrm{nM}$ and $50 \mathrm{nM}$ and transfected into cells with Dharmafect (5 $\mu \mathrm{L} /$ well; Dharmacon; GE Healthcare, USA). After determining the effective dose by qRT-PCR, gene expression and viability analysis were performed.

In the next step, in order to determine the synergistic effect of siRNA and Naloxone drug in cells, the initially determined effective doses of siRNA and Naloxone drug were given to the cells in combination. Cell viability analysis and qRT-PCR were performed to detect changes in gene expression levels of target genes.

The sequences of siRNA (Dharmacon; GE Healthcare, USA) were as follows (Table 1);

\section{Cell Viability Assay}

About $5 \times 10^{3}$ cells/well were seeded in 96 -well plates and incubated for 1 days. To determine the IC50 dose of Naloxone, PANC-1 cells exposed to a range of different Naloxone concentrations $(175-400 \mu \mathrm{M})$ for 24,48 and $72 \mathrm{~h}$. On the other hand, for siRNA viability analysis, experimental groups were divided into 
negative control and treated with different concentrations of VISTA-siRNA (5-50 nM) for $24 \mathrm{~h}$. Then, the cells were incubated by $20 \mu \mathrm{L}$ of MTT reagent (AppliChem, Germany) at $37^{\circ} \mathrm{C}$ for $35 \mathrm{~min}$ in the dark and analyzed by Nano Drop 2000 (Thermo Fisher Scientific, USA) at $570 \mathrm{~nm}$.

Cell viability was calculated by normalizing relative to the untreated control for Naloxone treatment and to the non-target siRNA treated group for siRNA treatment.

\section{Quantitative Real-time Pcr}

PANC-1 cells and HUVEC cells $\left(7 \times 10^{6}\right)$ were cultured in $75 \mathrm{~cm}^{2}$ flasks for 24 hours and then exposed to $271.789 \mu \mathrm{M}$ (IC50 dose) Naloxone drug for 72 hours. For siRNA application, were seeded in 6 well plates at a concentration of $2.5 \times 10^{4}$ cells per well and incubated for 24 hours.

Total RNA was used with trizol reagent (Sigma-Aldrich ${ }^{\circledR}$ ) and isolated from PANC-1 and HUVEC cells according to the manufacturer's protocol. $1 \mu \mathrm{g}$ of RNA was converted to cDNA using the RevertAid First Strand cDNA Synthesis Kit (Applied Biosystems, Thermo Fisher Scientific) on ice in the presence of a Random Hexamer primer. Gene expression studies were performed with SYBR Green Master Mix (Roche).

The following primers were used for gene expression analysis;

IRAK4:

F-5'-CGCTTCCTAGTTCGGCTGGTT-3'; R-5'-GTTTTGGGAACAGCATCTGCC-3'

VISTA:

F-5'-GATAGCGGCCTCTACTGCTG-3'; R-5'-TGGATGGTGCATCTTTGCCT-3'

TLR4:

F-5'-AGACCTGTCCCTGAACCCTAT-3'; R-5'-CGATGGACTTCTAAACCAGCCA-3' GAPDH:

F-5'-GGAGCGAGATCCCTCCAAAAT-3'; R-5'-GGCTGTTGTCATACTTCTCATGG-3'

The qRT-PCR cycling conditions were: initial denaturation at $95^{\circ} \mathrm{C}$ for $10 \mathrm{~min}$, followed by 40 cycles of denaturation at $95^{\circ} \mathrm{C}$ for $15 \mathrm{~s}$, annealing at $53^{\circ} \mathrm{C}$ for $30 \mathrm{~s}$ and extension at $72^{\circ} \mathrm{C}$ for $60 \mathrm{~s}$. All experiments were carried out in triplicate using a Quant Studio3 (Thermo Fisher Scientific, USA) Real-Time PCR instrument. Data analysis for Naloxone application was done with Qiagen RT ${ }^{2}$ Profiler PCR Data Analysis. For the siRNA application, it was normalized according to the Non-targeting siRNA samples and analyzed through the reference information and template of the manufacturer.

\section{Statistical Analysis}


Statistical analyses were performed by using "RT ${ }^{2}$ Profiler $^{\text {TM }}$ PCR Array Data Analysis" and graphs and statistical analysis were generated using GraphPad Prism software (version 8.0.1; GraphPad Software, Inc., La Jolla, CA, USA). Comparisons between treated groups and untreated controls were performed using the Student's t-test (two-tailed) or two-way ANOVA. The tests considered a basic significance level of $p<0.05$.

\section{Results}

TLR4 and VISTA gene expression levels are upregulated in pancreatic cancer compared to normal tissue.

According to data analysis in GEPIA 2 databases, a high level of gene expression of TLR4 $(p<0.05$, Fig. 1a) and VISTA ( $p<0.05$, Fig. 1c) proteins was observed in cancer tissues compared to normal tissue, while no significant difference was observed in the gene expression change of IRAK4 (Fig. 1b) protein. Comparable data were obtained with MTT and qRT-PCR.

\section{High level of gene expression of IRAK4 protein is associated with poor prognosis.}

According to the data analysis in the UALCAN database, TLR4 and IRAK4 gene expressions in pancreatic cancer are decreased in tumor tissue at different cancer stages compared to normal tissue. However, there was no data on VISTA gene expression from pancreatic cancer patients in the database.

Bioinformatic analysis showed no significant association between TLR4 ( $p<0.26$ Fig. 1d) gene expression and pancreatic cancer patient survival, whereas up-regulated IRAK4 ( $p<0.015$ Fig. 1e) gene expression predicted poor prognosis in pancreatic cancer patient survival.

\section{Effect of different concentrations and incubation times of Naloxone on PANC-1 cells proliferation}

The drug Naloxone dramatically suppressed the proliferation of PANC-1 cells in a concentration- and time-dependent manner. The half-maximum inhibitory concentration (IC50) was $271.789 \mu \mathrm{M}$ after 72 hours of Naloxone treatment. ( $p<0.01$; Fig. 1f).

\section{Effect of Naloxone treatment on TLR4, IRAK4 and VISTA gene expression in PANC-1 cells}

The effects of TLR4 antagonist Naloxone on TLR4, IRAK4 and VISTA were compared the level of gene expressions with $2^{-\triangle \Delta C T}$. According to the study, while TLR4 gene expression was significantly downregulated in PANC-1 cells compared to HUVEC cell, no statistically significant results were obtained in IRAK4 and VISTA gene expressions (Fig. 1g, Table 2).

Figure 1 Prognostic analysis of target genes in pancreatic cancer and effect of TLR4 inhibitor Naloxone on PANC-1. $\mathbf{a}, \mathbf{b}$ and $\mathbf{c}$ differential expression analysis of TLR4, IRAK4 and VISTA genes in pancreatic cancer ( 79 tumor cases and 171 normal cases). GEPIA2 generates a box plot showing gene expressions in pancreatic cancer and normal tissues (TCGA tumor versus TCGA normal + GTEx normal). UALCAN plot showing the patient-survival relationship of TLR4 $\mathbf{d}$ and IRAK4 e gene expressions. Blue line: lowexpression groups (132 cases) red line: high-expression groups (45 cases). $\mathbf{f}$ The effect of Naloxone drug 
applied to PANC-1 at different concentrations on cell viability e TLR4, IRAK4 and VISTA mRNA expression in PANC-1 and HUVEC cells following incubation with $271.789 \mu \mathrm{M}$ Naloxone

\section{Suppression Rates of GAPDH Gene Expression of 25 nM GAPDH-siRNA Transfection into a PANC-1 Cells}

24-hour 25 nM GAPDH-siRNA was applied to PANC-1 cells and gene expression levels were compared with the control group. As a result, a significant knockdown was observed in GAPDH gene expression compared to the control group ( $p<0.0001$; Fig. 2 a)

\section{VISTA-siRNA Transfection applied to PANC-1 Cells at Different Concentrations Causes Suppression of VISTA, TLR4 and IRAK4 Gene Expression.}

VISTA-siRNA and NT-siRNA were transfected into the PANC-1 cells at concentrations of 5, 10, 25, $50 \mathrm{nM}$. Gene expression results were normalized to the GAPDH gene. The siRNA dose that provided the maximum suppression was determined and used for further studies.

As a result of the application of $5 \mathrm{nM}$ VISTA-siRNA to the PANC-1 cells for 24 hours, no significant suppression was observed in VISTA, TLR4 and aIRAK4 gene expression compared to the control group.

However, as a result of the application of $10 \mathrm{nM}, 25 \mathrm{nM}$ and $50 \mathrm{nM}$ VISTA-siRNA to PANC-1 cells for 24 hours, a significant decrease in VISTA (Fig. 2b, c and d), TLR4 (Fig. 2e, f and g) and IRAK4 (Fig. 2h, i and j) gene expression was observed compared to the control group.

Figure 2 Effect of various siRNAs transfected into PANC-1 cells on expression of target genes aTransfection of $25 \mathrm{nM}$ GAPDH-siRNA into PANC-1 cells for 24 hours resulted in $96 \%$ suppression of GAPDH gene expression. VISTA gene expression was suppressed by $44 \%(p<0.01), 84 \%(p<0.001)$ and $96 \%$ ( $p<0.001)$, respectively, by transfection of b $10 \mathrm{nM}, \mathbf{c} 25 \mathrm{nM}$ and $\mathbf{d} 50 \mathrm{nM}$ VISTA-siRNA into PANC-1 cells for 24 hours. TLR4 gene expression was suppressed by 50\% $(p<0.05), 70 \%(p<0.01)$ and $75 \%$ $(p<0.01)$, respectively, by transfection of e $10 \mathrm{nM}, \mathbf{f} 25 \mathrm{nM}$ and $\mathbf{g} 50 \mathrm{nM}$ VISTA-siRNA into PANC-1 cells for 24 hours. IRAK4 gene expression was suppressed by 33\% ( $p<0.01), 60 \%(p<0.01)$ and $75 \%(p<0.001)$, respectively, by transfection of $\mathbf{h} 10 \mathrm{nM}, \mathbf{i} 25 \mathrm{nM}$ and $\mathbf{j} 50 \mathrm{nM}$ VISTA-siRNA into PANC-1 cells for 24 hours.

\section{Effect Of Sirna Transfection On Panc-1 Cells Viability}

MTT method was used as a viability analysis test to observe the effect of the VISTA gene on proliferation on the PANC-1 cells and to determine the effective dose on the cells. In the analyzes, different doses of VISTA-siRNA were studied in 5 replicates for each group. Absorbance values were measured at $570 \mathrm{~nm}$ with a spectrophotometer 24 hours after transfection and calculated with their mean values. The mean absorbance values of the siRNA-transfected cells were proportional to the mean absorbance values of the untransfected control cell group and the percentage of viability of the cells was calculated according to the siRNA doses. As a result, there was a significant decrease in viability in cells transfected with different concentrations of VISTA-siRNA compared to the control group (Fig. 3a). 
Naloxone and siRNA Combination Applications Create a Synergistic Effect on Target Genes in the PANC-1

Cells.

PANC-1 cells were treated with 24 hours of 25 nM VISTA-siRNA, 25 nM GAPDH-siRNA, and 25 nM NTsiRNA in combination with 72 hours of Naloxone. At the end of the combination application, the effects on VISTA, TLR4 and IRAK4 gene expressions were examined and statistically significant results were found.

As a result of the combination of 24-hour $25 \mathrm{nM}$ VISTA-siRNA and 72 hours Naloxone administration to PANC-1 cells, a significant knockdown was observed in VISTA, TLR4, and IRAK4 gene expression compared to the control (Fig. 3b).

\section{Effect of Naloxone and siRNA on PANC-1 Cells in Combination Applications on Cell Viability}

MTT viability analysis was performed to observe the effect of VISTA and TLR4 genes on cell proliferation by creating a synergistic effect on PANC-1 cells. PANC-1 cells were treated with 24-hour siRNA and 72 hours of Naloxone. In the analyzes, each group was studied with 3 replications. The averages of absorbance values measured at $570 \mathrm{~nm}$ wavelength of 3 wells were calculated. The mean absorbance values of the cells treated with Naloxone and siRNA were compared to the non-transfected control cells, and the percentage of viability was calculated. As a result, cell viability was significantly reduced when compared to the control group (Fig. 3c).

Figure 3. Enrichment analysis of differential expression genes associated with VISTA expression in pancreatic cancer a Dose dependence of cell viability activities $24 \mathrm{~h}$ after transfection with VISTA and NT siRNA into PANC-1 cells ( $p<0.001)$. The viability of PANC-1 cells treated with $5 \mathrm{nM}, 10 \mathrm{nM}, 25 \mathrm{nM}$, and 50 nM VISTA-siRNA, decreased to $85 \%, 81 \%, 79 \%$, and $76 \%$, respectively. The viability of cells treated with 25 GAPDH-siRNA decreased to 70\%. b Naloxone and 25nM VISTA-siRNA application in combination to PANC-1 cells showed suppression of $65 \%(p<0.001), 77 \%(p<0.001)$ and $74 \%(p<0.01)$ in VISTA, TLR4 and IRAK4 gene expressions, respectively. c MTT analysis graph of the effect of $25 \mathrm{nM}$ NT-siRNA $(p<0.05), 25$ nM GAPDH-siRNA $(p<0.01), 25 \mathrm{nM}$ VISTA-siRNA $(p<0.01)$ and combination treatment of Naloxone and 25 nM VISTA-siRNA $(p<0.01)$ application to PANC-1 cells on cell viability. The percentage of cell viability decreased to $98 \%, 71 \%$ and $79 \%$, respectively, in cells treated with 24-hour NT-siRNA, GAPDH-siRNA, and VISTA-siRNA compared to control cells. In cells treated with 72 hours of Naloxone and 24 hours of VISTAsiRNA, the percentage of cell viability decreased to $77 \%$ compared to control cells.

\section{Discussion}

PDAC is one of the most lethal solid malignancies and despite significant improvement in surgical techniques and postoperative results, the overall recurrence rate is approximately $85 \%$ and the 5 -year survival is less than $30 \%[3,12]$. 
Pancreatic cancer develops resistance to chemotherapy, radiotherapy and targeted therapies due to the complex tumor microenvironment, genetic and epigenetic changes [12]. For these reasons, more effective treatment strategies are needed. Immunotherapeutic approaches have emerged for the treatment of advanced malignancies. Recently, the focus has been on the development of drugs that target specific receptors on pancreatic cancer tumor cells and their use in combination with chemotherapeutic agents or inhibition of genes overexpressed by siRNA molecules [15].

In this study, Naloxone was applied to block TLR4, which is highly expressed in PANC-1 cell, in the first stage. Then, the IC50 dose was found, and it was used in the analysis -which is performed with qRT-PCR to detect the suppression of TLR4, IRAK4 and VISTA gene expressions. As a result, whereas a significant down-regulation was observed in TLR4 gene expression, no significant results were obtained in IRAK 4 and VISTA gene expressions. Human TLRs are expressed in pancreatic cancer tissue and many cancer cell lines, although they are little or not expressed in normal pancreatic cells $[15,16]$. TLR4 causes tumor progression by communicating with tumor cells, immune cells and tumor microenvironment. In many studies, TLR4 plays an important role in the inhibition of chemotherapy resistance and tumor formation [8]. In a study conducted in 2009, the effect of LPS on the invasive ability of PDAC was examined by targeting TLR4 and Myd88 with siRNA. As a result of the study, it was shown that LPS increases the invasive ability of PDAC and blockade of the NF-KB pathway reduces the increased invasive ability of LPS [16]. In a study by Hsiao et al. on hepatocellular carcinoma (HepG2) cells, it was observed that IL-6 upregulated LPS-induced TLR4. It was also determined that TLR4 activation increased proliferation, nitric oxide synthase (NOS) expression and NO production in HepG2 cells. In addition, it was observed that high chemoresistance was developed against antitumor drugs applied to HepG2 cells with very high TLR4 expression [17]. Long before TLR4 was discovered, Naloxone was found to block the biological activities of LPS, a component of the cell walls of gram-negative bacteria. Recently, it is known that Naloxone acts as TLR4 antagonist by binding to the LPS binding site of the MD2 protein [18].

In the second part of the study, the effect of siRNA silencing of VISTA, which is highly expressed in the PANC-1 cell, on gene expression of TLR4 and IRAK4 was investigated. VISTA-siRNA designed specifically for the VISTA gene and NT-siRNA were transfected into cells at 4 different concentrations by lipofection method. The effect of siRNA on VISTA was examined by qRT-PCR. Based on the qRT-PCR results, it has been observed that $24 \mathrm{nM}$ VISTA-siRNA application for 24 hours in PANC-1 human PANC-1 provides the most ideal suppression, since the combination will be applied in the optimizations and subsequent studies. The percentages of silencing in the cell were found to be statistically significant compared to control group, and moreoversilencing occurred when siRNAs were successfully transfected. qRT-PCR was performed to see the suppression of TLR4 and IRAK4 gene expressions in cells treated with VISTA-siRNA. After 24 hours of siRNA treatment at $25 \mathrm{nM}$ dose, suppression of IRAK4 and TLR4 gene expressions was found to be significant.

VISTA, which is one of the immune checkpoints and can act as both a ligand and a receptor, is known to suppress T cell proliferation and cytokine release. VISTA, which suppresses the T cell-associated response for immune avoidance and survival in many human cancers such as prostate cancer, non-small 
cell lung cancer (NSCLC), and colorectal carcinoma, is also highly expressed in CD68+ macrophages in pancreatic cancer $[19,20,21]$. Preclinical studies have shown that VISTA blockade releases T-cell effector functions and suppresses tumor growth in colon cancer models by synergizing with other immune checkpoint inhibitors such as anti programmed cell death-1 ligand1 (anti-PD-L1) [22].

Preclinical studies have shown that VISTA blockade releases T-cell effector functions and suppresses tumor growth in colon cancer models by synergizing with other immune checkpoint inhibitors such as anti-PD-L1 [22].

Patients with ovarian and endometrial cancer were treated with immune checkpoint inhibitors such as PD-1 blockade, but the results were not satisfactory for both ovarian(11,5-15\% overall) and endometrial canacers (about $13 \%$ overall) [20]. Therefore, the development of new treatment strategies, such as combined therapies, is anticipated to successfully treat patients resistant to anti-PD-1 therapy [22, 23].

As a result, both proteins were found to be associated with the NF-kB signaling pathway. However, little is known about the expression and clinical significance of VISTA in pancreatic cancer. Potential links between VISTA and TLR4 expression have yet to be discovered. Targeting the connections in the downstream signaling pathways of VISTA and TLR4 in pancreatic cancer will be beneficial for clinical studies on immune checkpoint inhibitors and oncogenic pathway inhibitors.

Since TLR4 is thought to have a synergistic effect in VISTA regulation, VISTA-siRNA and Naloxone were given together to PANC-1 cells. Cells were treated with an IC50 dose of Naloxone for the first 48 hours and VISTA-siRNA for the next 24 hours. VISTA, IRAK4 and TLR4 gene expressions were compared by qRT-PCR according to NT-siRNA applied cells. As a result of the study, a significant knockdown was detected in VISTA, TLR4 and IRAK4 gene expression levels when compared to the control group.

A greater suppression of VISTA gene expression was observed when treated with $25 \mathrm{nM}$ VISTA-siRNA alone compared to the combined treatment of Naloxone and VISTA-siRNA. Our hypothesize is; this is due to the fact of Naloxone and VISTA-siRNA may decrease the efficacy by negatively affecting each other in VISTA gene expression. Combination treatment of VISTA-siRNA with agents used in TLR4 inhibition into PANC-1 cells was found to significantly downregulate TLR4 and IRAK4 gene expressions which are located in its downstream pathway compared to VISTA-siRNA treatment alone. In addition, according to the results of MTT analysis, knockdown of VISTA and TLR4 caused a significant decrease in cell viability. Based on these results, the relationship between VISTA, TLR4 and IRAK4 molecule which are responsible for pancreatic cancer progression was determined at both gene expression and cell viability levels. The obtained data of the study show that TLR4 and IRAK4 molecule, which is involved in the TLR4 downstream pathway, are associated with poor prognosis in pancreatic cancer development and proliferation. Furthermore, TLR4 signaling pathway is associated with negative immune checkpoint VISTA in cancer development

As a result, it is thought that the existence of the relationship between the immune checkpoint VISTA and TLR4 will contribute to the development of anti-tumor studies via accepting these molecules as a 
therapeutic target and to better understand the signaling pathways and ligands regulated by VISTA.

\section{Conclusion}

In our study, we first used gene expression data using public databases to highlight the importance of the immune checkpoint and TLR4 in pancreatic cancer patients. In addition to the data, we have shown the critical importance of TLR4 signaling pathway and immune checkpoint VISTA in PDAC growth and proliferation with our analysis. It was concluded that the use of inhibitors designed for these proteins may be possible to halt the progression of cancer cells. Since TLR4 and VISTA signaling pathways are highly expressed in pancreatic cancer and are associated with different signaling pathways, potential connections with other signaling pathways need to be determinedwe think that immune checkpoint inhibitors may be an important marker in determining response in cancer therapy.

\section{Declarations}

Ethics approval and consent to participate This study was approved by the Pharmaceutical and NonMedical Research Ethical Committee of Necmettin Erbakan University, Meram Faculty of Medicine.

Consent for publication All authours consent to the publication of this study.

Availability of data and materials All data generated or analysed during this study are included in this published article.

Competing interests The authors declare no conflict of interest.

Funding This study was funded by Necmettin Erbakan University Scientific Research Projects Coordination Unit, Project number 191315002.

Authors' contributions E.N.K conceptualized the project; E.N.K., N.K and K.S.B.T designed experiments; E.N.K., K.S.B.T and T.D. performed experiments; E.M. analyzed data; K.S.B.T wrote original manuscript draft; E.M. wrote revised manuscript draft; E.N.K. supervised and administered the project.

Acknowledgements Cell culture assays were performed at KTO University.

Disclosure of potential conflicts of interest Not applicable

Research involving Human Participants and/or Animals Not applicable

Informed consent Not applicable

\section{References}

[1] F. H. Demirelli, “Kanserin Moleküler Genetik Temelleri," Güncel Klin. Onkol. Sempozyum Dizisi, no. 37, pp. 9-15, 2003. 
[2] H. Sung et al., "Global Cancer Statistics 2020: GLOBOCAN Estimates of Incidence and Mortality Worldwide for 36 Cancers in 185 Countries," CA. Cancer J. Clin., vol. 71, no. 3, pp. 209-249, May 2021.

[3] P. Rawla, T. Sunkara, and V. Gaduputi, “Epidemiology of Pancreatic Cancer: Global Trends, Etiology and Risk Factors," World J. Oncol., vol. 10, no. 1, pp. 10-27, 2019.

[4] K. Banerjee et al., "Emerging trends in the immunotherapy of pancreatic cancer," Cancer Lett., vol. 417, pp. 35-46, 2018.

[5] J. L. Lines et al., "VISTA is an immune checkpoint molecule for human T cells," Cancer Res., vol. 74, no. 7, pp. 1924-1932, 2014.

[6] C. Hashimoto, K. L. Hudson, and K. V. Anderson, "The Toll gene of drosophila, required for dorsalventral embryonic polarity, appears to encode a transmembrane protein," Cell, vol. 52, no. 2, pp. 269-279, 1988.

[7] B. Guven and M. Can, "Role Of Toll Like Receptors In Various Diseases," Sak. Med. J., vol. 2, no. 1, pp. $1-10,2012$.

[8] C.-Y. Chen, C.-L. Kao, and C.-M. Liu, "The Cancer Prevention, Anti-Inflammatory and Anti-Oxidation of Bioactive Phytochemicals Targeting the TLR4 Signaling Pathway.," Int. J. Mol. Sci., vol. 19, no. 9, p. 2729, Sep. 2018.

[9] X. Chen, L. S. Mangala, C. Rodriguez-Aguayo, X. Kong, G. Lopez-Berestein, and A. K. Sood, "RNA interference-based therapy and its delivery systems," Cancer Metastasis Rev., 2018.

[10] F. Bu et al., "Expression profile of gins complex predicts the prognosis of pancreatic cancer patients," Onco. Targets. Ther., vol. 13, pp. 11433-11444, 2020.

[11] D. S. Chandrashekar et al., "UALCAN: A Portal for Facilitating Tumor Subgroup Gene Expression and Survival Analyses," Neoplasia (United States), vol. 19, no. 8, pp. 649-658, 2017.

[12] R. Casolino et al., "Reshaping preoperative treatment of pancreatic cancer in the era of precision medicine," Ann. Oncol., vol. 32, no. 2, pp. 183-196, 2021.

[13] Y. Sunami and J. Kleeff, "Immunotherapy of pancreatic cancer," in Progress in Molecular Biology and Translational Science, 2019.

[14] M. Aslan, R. Shahbazi, K. Ulubayram, and B. Ozpolat, "Targeted Therapies for Pancreatic Cancer and Hurdles Ahead.," Anticancer Res., vol. 38, no. 12, pp. 6591-6606, Dec. 2018.

[15] J. Vaz and R. Andersson, "Intervention on toll-like receptors in pancreatic cancer," World J. Gastroenterol., vol. 20, no. 19, pp. 5808-5817, 2014. 
[16] M. A. Lanki et al., "Toll-like receptor 2 and Toll-like receptor 4 predict favorable prognosis in local pancreatic cancer," Tumor Biol., vol. 40, no. 9, pp. 1-9, 2018.

[17] M. Ikebe et al., "Lipopolysaccharide (LPS) increases the invasive ability of pancreatic cancer cells through the TLR4/MyD88 signaling pathway," J. Surg. Oncol., vol. 100, no. 8, pp. 725-731, Dec. 2009.

[18] C. C. Hsiao et al., "Toll-like receptor-4 is a target for suppression of proliferation and chemoresistance in HepG2 hepatoblastoma cells," Cancer Lett., 2015.

[19] X. Wang, Y. Zhang, Y. Peng, M. R. Hutchinson, K. C. Rice, and H. Yin, “Pharmacological characterization of the opioid and (+ ) -naloxone as antagonists of toll-like receptor 4 Tables of Links," 2016.

[20] J. Blando et al., "Comparison of immune infiltrates in melanoma and pancreatic cancer highlights VISTA as a potential target in pancreatic cancer," Proc. Natl. Acad. Sci. U. S. A., vol. 116, no. 5, pp. 16921697, 2019.

[21] X. Huang et al., "VISTA: an immune regulatory protein checking tumor and immune cells in cancer immunotherapy," J. Hematol. Oncol., vol. 13, no. 1, p. 83, 2020.

[22] Postow, M. A., Callahan, M. K. \& Wolchok, J. D. Immune checkpoint blockade in cancer therapy. J. Clin. Oncol. 33, 1974-1982 (2015).

[23] Ott, P. A. et al. Safety and antitumour activity of pembrolizumab in advanced programmed death ligand 1-positive endometrial cancer: results from the KEYNOTE-028 Study. J. Clin. Oncol. 35, 2535-2541 (2017).

\section{Tables}

Table 1. siRNA sequence information in the VISTA, NT ve GAPDH siRNA-pool.

a VISTA; ON-TARGETplus Human EGFR (1956) siRNA - SMARTpool, $5 \mathrm{nmol}$ (Dharmacon, GE Healthcare, L-032651-02-005) b Negative control; ON-TARGETplus Human Non-targeting Pool, 5 nmol (Dharmacon, GE Healthcare, D-001810-10-05) c GAPDH; ON-TARGETplus Human GAPDH Control Pool, 5 nmol (Dharmacon, GE Healthcare, D-001830-10-05)

\section{a}


Sequence

1. siRNA sequence

ON-TARGETplus SMARTpool siRNA J-032651-21, VISTA GUGAGACAGUGCCGAGAUA

Mol. Wt: 13,445.1 (g/mol) Ext. Coeff: 367,303 (L/mol.cm)

2. siRNA sequence ON-TARGETplus SMARTpool siRNA J-032651-22, VISTA CCACAAUGCUUGAUGCCAG

Mol. Wt: 13,445.1 (g/mol) Ext. Coeff: 361,073 (L/mol.cm)

3. siRNA sequence ON-TARGETplus SMARTpool siRNA J-032651-23, VISTA GGACAAAGGGCACGAUGUG

Mol. Wt: 13,460.1 (g/mol) Ext. Coeff: 359,738 (L/mol.cm)

4. siRNA sequence ON-TARGETplus SMARTpool siRNA J-032651-24, VISTA CGGUGGAACUACUACGACUAAA

Mol. Wt: 13,430.1 (g/mol) Ext. Coeff: 373,622 (L/mol.cm)

\begin{tabular}{|lr|}
\hline Sequence & Sequence information \\
\hline 1. siRNA sequence & UGGUUUACAUGUCGACUAA \\
\hline 2. siRNA sequence & UGGUUUACAUGUUGUGUGA \\
\hline 3. siRNA sequence & UGGUUUACAUGUUUUCUGA \\
\hline 4. siRNA sequence & UGGUUUACAUGUUUUCCUA \\
\hline
\end{tabular}

\begin{tabular}{|ll|}
\hline Sequence & Sequence information \\
\hline 1. siRNA sequence & GUCAACGGAUUUGGUCGUA \\
\hline 2. siRNA sequence & CAACGGAUUUGUCGUAUU \\
\hline 3. siRNA sequence & GACCUCAACUACAUGGUUU \\
\hline 4. siRNA sequence & UGGUUUACAUGUUCCAAUA \\
\hline
\end{tabular}


Table 2 Comparison of mean $2^{-\triangle \Delta C T}$ and P-value of TLR4, IRAK4 and VISTA gene expression in PANC-1 and HUVEC cells following incubation with $271.789 \mu \mathrm{M}$ of Naloxone.

\begin{tabular}{lllllll} 
& VISTA & & TLR4 & \multicolumn{3}{c}{ IRAK4 } \\
\hline & $2^{-\triangle \Delta C T}$ & $P$ & $2^{-\Delta \Delta C T}$ & $P$ & $2^{-\Delta \Delta C T}$ & $P$ \\
\hline HUVEC & 1,82 & 0,65 & 0,485 & 0,001 & 0,91 & 0,49 \\
\hline PANC-1 & 0,56 & 0,02 & 0,00585 & 0,001 & 0,045 & 0,001
\end{tabular}

Figures 

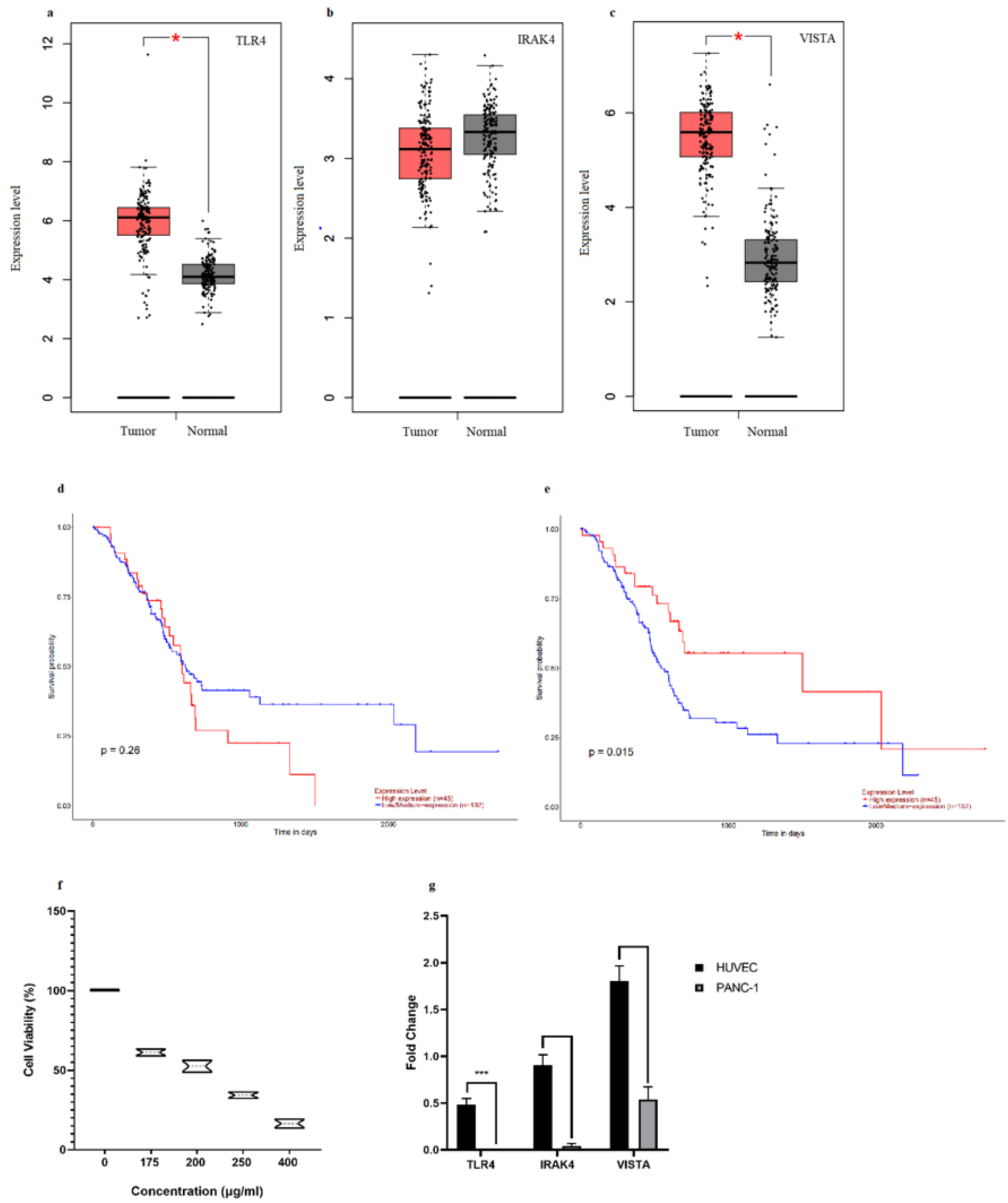

\section{Figure 1}

Prognostic analysis of target genes in pancreatic cancer and effect of TLR4 inhibitor Naloxone on PANC1. $a, b$ and $c$ differential expression analysis of TLR4, IRAK4 and VISTA genes in pancreatic cancer (79 tumor cases and 171 normal cases). GEPIA2 generates a box plot showing gene expressions in pancreatic cancer and normal tissues (TCGA tumor versus TCGA normal + GTEx normal). UALCAN plot showing the patient-survival relationship of TLR4 $d$ and IRAK4 e gene expressions. Blue line: low- 
expression groups (132 cases) red line: high-expression groups (45 cases). $f$ The effect of Naloxone drug applied to PANC-1 at different concentrations on cell viability e TLR4, IRAK4 and VISTA mRNA expression in PANC-1 and HUVEC cells following incubation with $271.789 \mu \mathrm{M}$ Naloxone
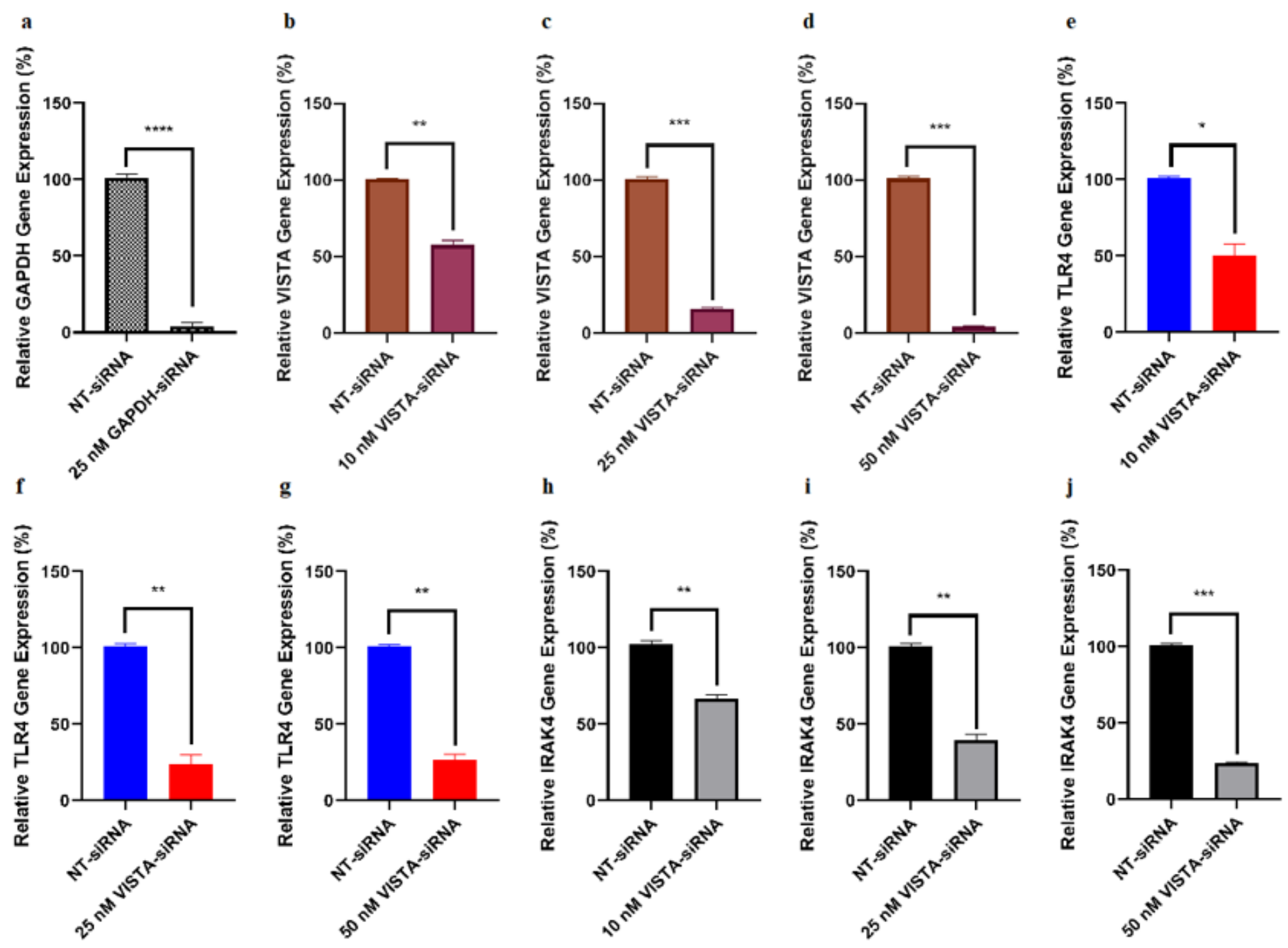

Figure 2

Effect of various siRNAs transfected into PANC-1 cells on expression of target genes aTransfection of 25 nM GAPDH-siRNA into PANC-1 cells for 24 hours resulted in 96\% suppression of GAPDH gene expression. VISTA gene expression was suppressed by $44 \%(p<0.01), 84 \%(p<0.001)$ and $96 \%(p<0.001)$, respectively, by transfection of b $10 \mathrm{nM}, \mathrm{c} 25 \mathrm{nM}$ and d $50 \mathrm{nM}$ VISTA-siRNA into PANC-1 cells for 24 hours. TLR4 gene expression was suppressed by $50 \%(p<0.05), 70 \%(p<0.01)$ and $75 \%(p<0.01)$, respectively, by transfection of e $10 \mathrm{nM}$, f $25 \mathrm{nM}$ and g $50 \mathrm{nM}$ VISTA-siRNA into PANC-1 cells for 24 hours. IRAK4 gene expression was suppressed by $33 \%(p<0.01), 60 \%(p<0.01)$ and $75 \%(p<0.001)$, respectively, by transfection of $h 10 \mathrm{nM}, \mathrm{i}$ $25 \mathrm{nM}$ and $\mathrm{j} 50 \mathrm{nM}$ VISTA-siRNA into PANC-1 cells for 24 hours. 


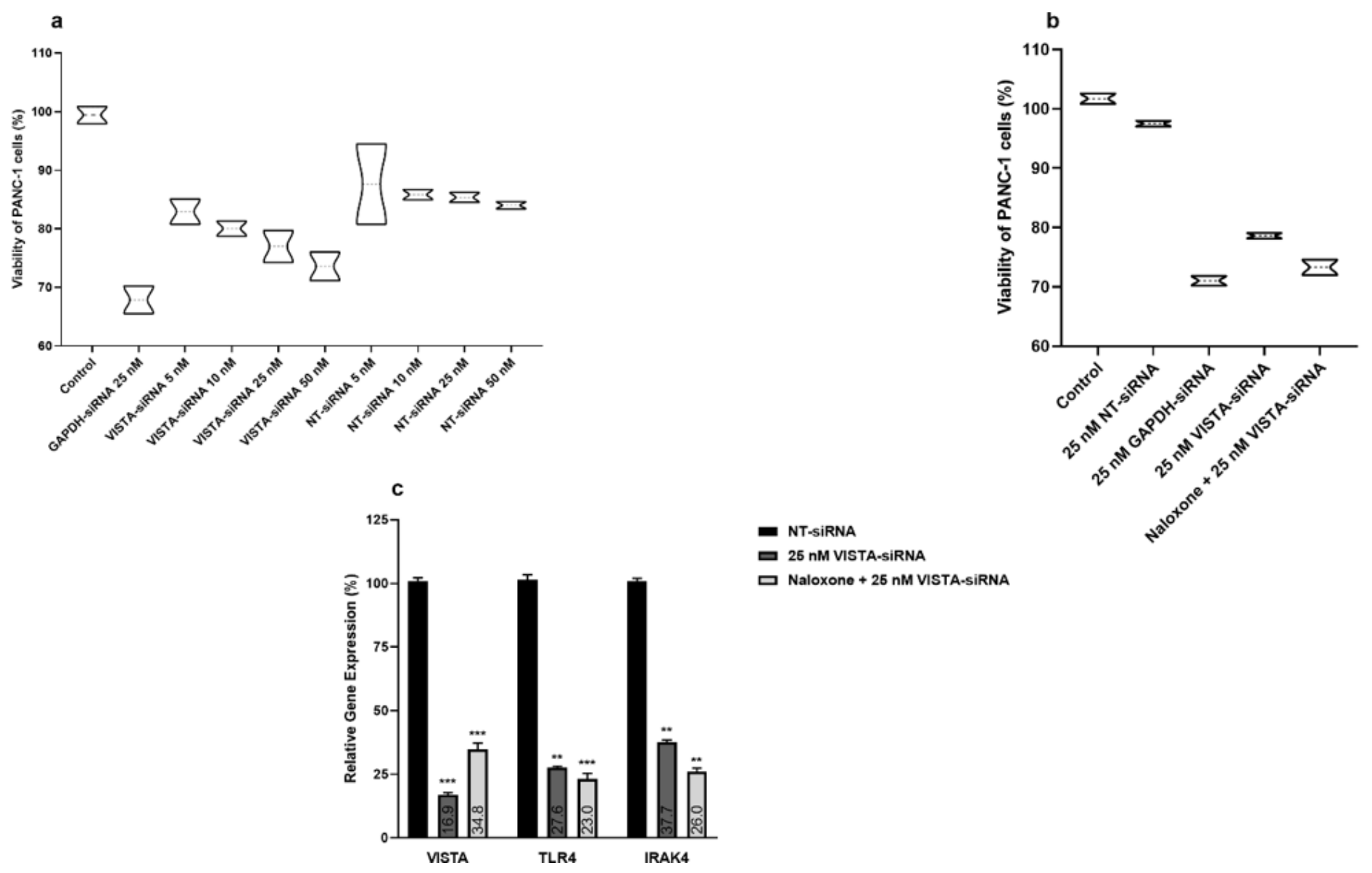

Figure 3

Enrichment analysis of differential expression genes associated with VISTA expression in pancreatic cancer a Dose dependence of cell viability activities $24 \mathrm{~h}$ after transfection with VISTA and NT siRNA into PANC-1 cells $(p<0.001)$. The viability of PANC- 1 cells treated with $5 \mathrm{nM}, 10 \mathrm{nM}, 25 \mathrm{nM}$, and $50 \mathrm{nM}$ VISTAsiRNA, decreased to $85 \%, 81 \%, 79 \%$, and $76 \%$, respectively. The viability of cells treated with $25 \mathrm{GAPDH}$ siRNA decreased to $70 \%$. b Naloxone and 25nM VISTA-siRNA application in combination to PANC-1 cells showed suppression of $65 \%(p<0.001), 77 \%(p<0.001)$ and $74 \%(p<0.01)$ in VISTA, TLR4 and IRAK4 gene expressions, respectively. c MTT analysis graph of the effect of $25 \mathrm{nM}$ NT-siRNA $(\mathrm{p}<0.05), 25 \mathrm{nM} \mathrm{GAPDH}-$ siRNA ( $p<0.01), 25 \mathrm{nM}$ VISTA-siRNA $(p<0.01)$ and combination treatment of Naloxone and $25 \mathrm{nM}$ VISTAsiRNA $(p<0.01)$ application to PANC-1 cells on cell viability. The percentage of cell viability decreased to $98 \%, 71 \%$ and $79 \%$, respectively, in cells treated with 24-hour NT-siRNA, GAPDH-siRNA, and VISTA-siRNA compared to control cells. In cells treated with 72 hours of Naloxone and 24 hours of VISTA-siRNA, the percentage of cell viability decreased to $77 \%$ compared to control cells. 